\title{
Computation of Liquid Crystal Display Device Parameters Through Image Analysis
}

\author{
${ }^{*}$ S. Sreehari Sastry ${ }^{a^{*}}$, M. Sailaja ${ }^{a}$ S. Lakshminarayana ${ }^{b}$ and S.T. $\mathrm{Ha}^{\mathrm{c}}$ \\ ${ }^{a}$ Dept. of Physics, Acharya Nagarjuna University, Nagarjunanagar, Andhra Pradesh, India \\ ${ }^{b}$ Department of Electronics and Communication Engineering, K.L. University Vaddeswaram, \\ Guntur, Andhra Pradesh, India \\ ${ }^{c}$ Department of Chemical Science, Faculty of Science, Universiti Tunku Abdul Rahman, \\ JalanUniversiti, Kampar, Perak, Malaysia \\ Corresponding author: *S. Sreehari Sastry
}

\begin{abstract}
:
It has become a challenging problem now a days to select a liquid crystal material best suited for electro-optical display. Image analysis technique in conjunction with polarizing optical microscope is proposed to determine display device optical parameters for homogeneously aligned functional materials on chiral ester liquid crystals ((S)-(-)-2-methylbutyl4-(4- $n$-alkanoyloxybenzoyloxy) biphenyl-4-carboxylates (where $n=16$ and 18)). The textures are recorded as a function of temperature dependence of optical parameters like Luminance, Luminance uniformity, Luminance contrast ratio, Luminance modulation, Gray Shades, Visual Acuity, and Color Saturation that are computed for these functional materials from the series of images recorded texture using MATLAB software. These parameters are useful for the selection liquid crystal material for choosing best electro-optic display devices. This is a new and simple technique computing different optical parameters of functional materials in specific for ferroelectric liquid crystals, without using various instrumental methods. This method of evaluation of optical parameters for various liquid crystal compounds facilitates to identify the selectivity of specific liquid crystal compound suitability to the best of optical display.
\end{abstract}

Keywords: Image Liquid crystals; image analysis; display device optical parameter, luminance

\section{INTRODUCTION}

Liquid crystal displays (LCDs) play a vital role in entertainment field, medical field etc., as it has adopted a means of displaying information. Compare to traditional LCDs, Ferroelectric liquid crystal (FLC) displays are much more advantageous as it possesses spontaneous polarization, rapid response characteristics which is essential in eliminating video anomalies, very wide viewing angles, enabling implementation of high resolution (with more deduced pixel size), preventing crosstalk due to strong interactions between molecules [1]. High resolution and sensitive experimental techniques are now available to record the transformations which occur during the mesophase changes. For characterization of liquid crystalline properties it is necessary to identify the transition phases and transition temperatures [2-4]. The study on ferroelectric liquid crystals is generating much interest for synthesizing and characterizing the new FLCs as it possesses as wide applications in display devices. [5-9]. Having these objectives, the present attempt is aimed to study the transition temperatures of the two synthesized ferroelectric liquid crystals
((S)-(-)-2-methylbutyl4-(4- $n$-alkanoyloxy benzoyloxy) biphenyl-4-carboxylates (where $n=16$ and 18)) through the image analysis techniques, and the statistical and image moments approaches applied to the microscopic textures of FLCs. On par with transition temperatures for FLC textures, it is demonstrated here through image analysis to compute the electro optical device luminance and its dependent parameters such as luminance contrast ratio, luminance contrast modulation, luminance uniformity, gray shades, visual acuity, and blending weight. The results of the study are compared with those of published earlier [10]. Similar work was done previously for room temperature liquid crystals, cholesteric liquid crystals, and for discotic liquid crystals $[11,12,13,14]$. through image analysis on FLC textures at various transition temperatures. Luminance power describes the amount of luminous power detected by an eye while looking at a surface at a particular angle. Hence, it indicates the brightness of a surface. Luminance is applied in video industry to set the overall brightness of displays. For ideal optical systems, luminance output is equal to input luminance. This means that image of any object cannot be brighter than the object. 
Luminance measurement can be done using a light meter. Light meter is used in various fields like photography, cinematography, scenic design. It is necessary to measure the luminance of modern liquid crystal displays. There are various types and sizes of displays. Various methods are chosen for implementing the maximum luminance at very low power consumption. In general, many measuring points are necessary for the determination of luminance ratios for the entire LCD screen. To get the value of luminance, a point-by-point measurement has to be performed by the luminance meter which is time consuming. For screens of having a more number of pixels per unit area in modern high resolution ultra high definition (UHD) devices, the measurement of small details is not possible with a luminance meter. The image resolving measurement method is adapted to the luminance using high resolution digital camera. Luminance and its dependent parameters vary in different electro optic devices. Luminance value increases with white surface, whereas for black surface it is decreased. The entire information pertaining to the luminance and its dependent parameters are recorded instantaneously on liquid crystal textural images [15-16].

\section{Materials and Methods:}

\section{EXPERIMENTAL}

The chemical composition of the chosen liquid crystals of chiral esters is (S)-(-)-2-methylbutyl 4'(4"-n-alkonoyloxy benzoy loxy) biphenyl-4carboxylates with $n=16$ and $n=18$, showing variant alkyl chain lengths. These are synthesized and used in indium tin oxide (ITO) coated homogeneous (planar) cells having an area of $5 \mathrm{~mm} \times 5 \mathrm{~mm}$ area with $8.7 \mu \mathrm{m}$ spacing (tolerance is $\pm 0.2 \mu \mathrm{m}$ ). The cells are obtained from Instec Inc., USA. The chiral ester mesogens are injected into liquid crystal cell through capillary action by heating the sample to the isotropic state. Different phase transitions ae able to be observed with the naked eye during the time of cooling of the liquid crystal cell after injecting the samples, and a good alignment has formed due to very slow cooling down the cell. The flow direction of the liquid crystal during cell filling appears to influence the molecular alignment in the resultant liquid crystal layer. The nature of the alignment surface in the cells will obviously control the appearance. Polarizing optical microscope (Meopta) is used to observe the respective transformation phases of sample textures simultaneously and instantaneously as a function of temperature with its auxiliary attachments, such as hot stage and a high resolution digital camera. The observations are recorded by a high resolution Cannon EOS REBELXS/EOS1000D camera with 10.10 megapixel image sensor [17]. The colour image has a resolution of $2816 \times 1880$ pixels which represents 24 bit true colour pixel tone in total and the intensity values range from 0 to 255 in each red $(\mathrm{R})$, green $(\mathrm{g})$, blue (B) colours. However, the translated gray scale image is used here for analysis. The size of images are selected for 256. The program has been coded using MATLAB software for analysis of textures which is an efficient tool for computational analysis [18-19] Theory.

Application of computational techniques and algorithms on image data is quite useful for finding the various parameters of LCD's. Hence image analysis is a good method to extract the information of images. In the current article, various significant parameters of the LCD's such as luminance, luminance contrast ratio, luminance uniformity, contrast modulation, gray shades and visual acuity are measured with computational techniques at various liquid crystal textures. An image or texture $I(i, j)$ is of size $m$-by- $n$ is a two dimensional function composed of $m$ pixels in the vertical direction and $n$ pixels in the horizontal direction, $i, j$ are horizontal and vertical coordinates of the image. The total number of pixels in the image is $m * n=N, 0 \leq i \leq m, 0$ $\leq j \leq n$ [17]. The defined statistical parameters are explained below.

Luminance (L): It is the intensity of light emitted from a surface per unit area in a given direction. It is also known as luminance signal of a composite television signal which carries information on the brightness of the image. Luminance is often used to characterize emission or reflection from flat, and diffuse surfaces. The luminance indicates the amount of luminous power that is detected by an eye looking at the surface from a particular angle of view. A luminance level equal or higher than 85 candel as per square meter is the minimum threshold level recommended. [20]. Luminance variability is assessed under given (nominally) uniform, full screen images with gray levels from black (i.e., $\mathrm{RGB}=0,0$, 0) to white (i.e., $\mathrm{RGB}=255,255,255$ )

$L=0.2989 * \frac{1}{N} \sum_{i=1}^{m} \sum_{j=1}^{n} \operatorname{IR}(i, j)+$

$0.587 * \frac{1}{N} \sum_{i=1}^{m} \sum_{j=1}^{n} I B(i, j)+0.1140 *$

$\frac{1}{N} \sum_{i=1}^{m} \sum_{j=1}^{n} I G(i, j)$

\section{Where}

$I_{R}=$ intensity of Red light in image.

$\mathrm{I}_{\mathrm{B}}=$ intensity of Green light in image.

$\mathrm{I}_{\mathrm{G}}=$ intensity of Blue light in image.

Luminance Uniformity (LU):It is defined as the ratio of minimum luminance to maximum luminance. It is a measure of constancy of luminance level across the active area of display [21]. Luminance uniformity(LU) provides good screen perception [22] A screen should appear to have uniform brightness across the screen face. For 
uniform display, the luminance averages across 1 degree area at the minimum design viewing distance. Luminance should not vary from one area of the display to another by more than 1.7:1 [23]

$$
L U=\frac{\left(L_{\text {max }}-L_{\text {min }}\right)}{L_{\text {avg }}} \times 100 \ldots
$$

Where

$$
\begin{aligned}
& \mathrm{L}_{\max }=\text { Maximum value of luminance. } \\
& \mathrm{L}_{\min }=\text { Minimum value of luminance. }
\end{aligned}
$$

\section{Luminance contrast ratio (LCR):}

LCR is another major determinant of perceived picture quality. If a picture has high LCR, it is judged to be sharper and crisper than a picture with lower LCR. Contrast is created by the difference in luminance, i.e.., the amount of reflected light, reflected from two adjacent surfaces. Contrast is important in systems that automate the selection of colours. Itinerates is ? the degree of difference of an LCD monitor's ability to produce bright whites and the dark blacks. It is the ratio of luminance between the brightest white and the darkest black that can be produced. When the darker surface is black, it reflects no light, hence the ratio is 1 . Contrast is usually expressed as percentage, (and then the ratio is multiplied by 100). The maximum contrast is thus $100 \%$. Contrast ratio is a quantity intended to correlate with the perceived brightness contrast, usually defined by one of a number of formulae (see below) which involve Luminance [24]. Greater screen brightness is achieved with a contrast ratio greater than 5:1. In outdoor environments under the shade, such a display can provide an excellent image quality. Contrast ratio (CR) is the ratio of luminance between the brightest "white" and the darkest "black" that can be produced on a display.

Luminus Contrast Ratio (LCR)

$$
=\frac{\left(\boldsymbol{L}_{\max }-\boldsymbol{L}_{\min }\right)}{\left(\boldsymbol{L}_{\min }\right)}
$$

Where $\mathrm{L}_{\max }=$ Maximum value of luminance $\mathrm{L}_{\min }=$ Minimum value of luminance

With $1 \leq \mathrm{CR} \leq \infty \quad \mathrm{CR}=1$ means no contrast

Luminance contrast modulation (LCM): Peak-to-Peak Contrast (Michelson Contrast, Modulation) measures the relation between the spread and the sum of two luminance values. This definition is typically used in signal processing theory, to determine the quality of a signal relative to its noise level. In the context of vision, such noise could be caused by scattered light introduced into the view-path by a translucent element partly obscuring the scene behind it [25-28]

Luminus Contrast Modulation

$$
=\frac{\left(L_{\text {max }}-L_{\text {min }}\right)}{\left(L_{\max }+\boldsymbol{L}_{\min }\right)}
$$

$\mathrm{L}_{\max }=$ Luminance on the lighter surface
$\mathrm{L}_{\min }=$ Luminance on the darker surface

Grey shades: Based on the idea of brightest areas being white and the darkest areas being black, brightness levels between the two extremes are referred to as gray levels or shades and the ability to display them is termed as gray scale. The number of gray scales is determined by contrast level and ability of human visual perception. Our visual system reacts to the changes in brightness level as a logarithmic function. Hence very small difference in brightness may not be perceived. The number of grey shades (G) that can be displayed can be defined as a logarithmic function based on contrast ratio (G) [2930]

$$
G=1+(1 / \log (\sqrt{ } 2)) * \log \left(L_{\max } / L_{\text {min }}\right) \quad \ldots(5)
$$

Visual acuity: Visual system special resolution is expressed by Acuity. It can also be seen as sharpness which is measured in cycles per visual degree. Human vision performance depends on luminance [31]. Under low brightness conditions, acuity drops significantly and spatial detail is lost even for healthy observers. The luminance is not constant over the whole image, but may change locally [32]. The following relation determines the luminance to maximal resolvable frequency from Shaler's data:

$$
\begin{gathered}
f(L)=25.72+ \\
\text { 0.35) } \ldots \ldots(6)
\end{gathered}
$$

Color saturation: Saturation determines the color closeness to gray values. The pure color has saturation equal to 1 and the gray value has the saturation equal to 0

$$
\begin{array}{r}
\text { Saturation: } 1-\frac{\min \{R, G, B\}}{\text { Intensity }} \quad \ldots .(7) \\
\text { where intensity }=\frac{R+G+B}{3}
\end{array}
$$

\section{RESULTS AND DISCUSSION}

With the help of Polarizing Microscope, (POM) liquid crystal textures of the sample ((S)-(-)-2methylbutyl 4_-(4_-_n-al-kanoyloxybenzoyloxy) biphenyl-4-carboxylates (where $n=16$ and18)) as a function of temperature were recorded at the heating/cooling rate of $0.2^{\circ} \mathrm{C}$ per minute from the solid phase of the sample to isotropic phase (I) on heating and vice versa on cooling. During this process the FLC samples underwent phase transitions from crystalline phase to the isotropic phase via smectic- $C^{*}$, smectic-A, on heating and vice versa on cooling. While cooling two more sub phases such as crystal $1(\mathrm{Cr} 1)$, crystal $2(\mathrm{Cr} 2)$ were observed, that is 
smectic-A, smectic-A- smectic-C, smectic-C ${ }^{*}-\mathrm{Cr} 2$, $\mathrm{Cr} 2-\mathrm{Cr} 1$. Transition temperatures of the two chiral ferroelectric liquid crystals are shown below and in good agreement with ref. [10].

Heating cycle:

$\mathrm{Cr} 162^{0} \mathrm{C} \rightarrow \mathrm{SmC}^{*} 112^{0} \mathrm{C} \rightarrow \mathrm{SmA} 153^{\circ} \mathrm{C} \rightarrow \mathrm{I}$

Cooling cycle:

$\mathrm{Cr} 151^{\circ} \mathrm{C} \leftarrow \mathrm{Cr} 260^{\circ} \mathrm{C} \leftarrow \mathrm{SmC}^{*} 101.5^{\circ} \mathrm{C} \leftarrow \mathrm{SmA}$

$152.5^{\circ} \mathrm{C} \leftarrow \mathrm{I}$

[ Cr1, Crystal 1; Cr2, Crystal 2; SmC*,

SmecticC $*$ :SmA, SmecticA; I, Isotropic]

The experiment was done for both heating and cooling cycles. Plots are drawn for only cooling cycles to avoid the paramorphic defects. Computed parameter behavior variations were observed for different FLCs during plotting and its significant variations in phases were shown in Figure-1 to Figure-7 .These transition in phases as a function of temperature leads to changes in textural features during heating and cooling. These are more perceptible at the phase transitions. Even a small variation in the texture leads to a change in transmitted light intensity. Required parameters are computed using MATLAB software. Hence present methodology is highly accurate for the identification of phase transitions of liquid crystals and to compute the various luminance parameters [11-14].

Luminance is calculated as weighted summation of intensities over spectral range (blue to red) covered by human vision from equation 1 . The importance of luminance is enhanced by the fact that the human mind will react more positively to the bright illuminated scenes and objects. Users are typically more drawn to brighter displays because there are more pleasing to the eye and for easy to read. The higher end of temperature range is known as clearing point, above which, the liquid crystal loses its birefringence properties. Usually the crossed polarizers will not allow any light to pass through and creates dark field. When the LCD is cooled down to below its clearing point, the display will work again. The temperatures at the clearing point vary greatly from material to material. Figure 1 shows the graph drawn for luminance (L) as a function of Temperature. These measurements are

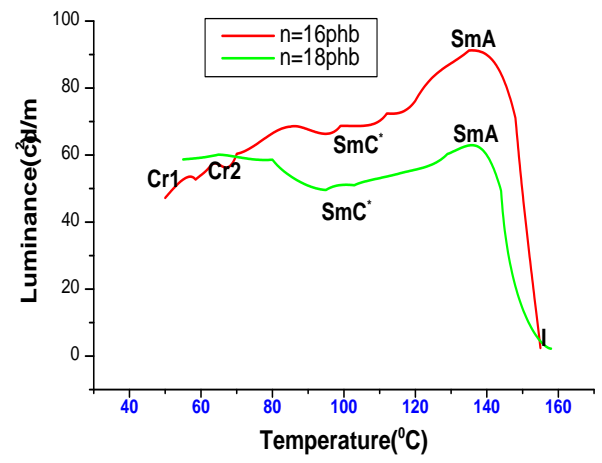

Fig: 1. Temperature dependence of luminance for $\mathrm{n}=16$ and 18

made in the phase transition regions between $\mathrm{SmC}^{*}-$ SmA-I phase of the sample (Ferroelectric phase region). Luminance values are higher in $\mathrm{n}=16$ compare to $\mathrm{n}=18$. The $\mathrm{L}$ for $\mathrm{n}=16$ and 18 , as shown in Fig. 1 increases with increasing temperature till the smectic-A phase and suddenly drops down to isotropic phase without exhibiting any intermediate value.

The significance of luminance uniformity can be viewed in the context of brightness perception. The uniformity in brightness, luminance gradient, change in the overall luminance level and frequency in occurrence of maximum and minimum luminance effect brightness perception and will influence the effect of luminance uniformity. Luminance uniformity (LU) versus temperature (which is shown in fig. 2) is calculated from equation-2. There is

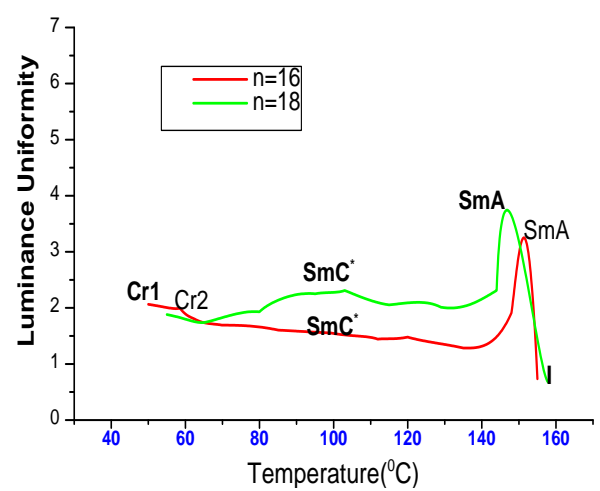

Fig. 2. Temperature dependence of luminance uniformity for $n=16$ and 18

A sudden shoot up of LU for both $n=16$ and $\mathrm{n}=18$, at $\mathrm{SmA}$ the LU is almost stable during $\mathrm{SmC}^{*}$ phase. Average LU value of 1.5 is maintained with $\mathrm{n}=16$ and for $\mathrm{n}=18$ it is around 2.5. Contrast Ratio is calculated from equation 3 and variation is observed from graph 3. There is an uniform decrease in Fig.3. Temperature dependence of contrast ration luminance for $n=16$ and 18temperature for $n=16$ and $18 \mathrm{CR}$ value for $n=16$ in $C^{*}$ phase. After $140^{\circ} \mathrm{C}$ there is a sudden shoot up in CR until Smectic-A phase has 
reached. There is uniform increase of contrast ratio for the material with $n=18$ compound. Hence, the $\mathrm{n}=18$ compound is more preferable relatively to $\mathrm{n}=16$ for better contrast ratio. The comparison of weber contrast with the $\mathrm{CR}$ indicates that there is only one unit difference in their values. Both the samples are having contrast ratio greater than standard values for better human eye perception.

Luminance Contrast modulation (LCM) is calculated from equation 4 and its variation with respect to temperature is observed in graph 4 . In both the samples LCM variation is almost uniform during

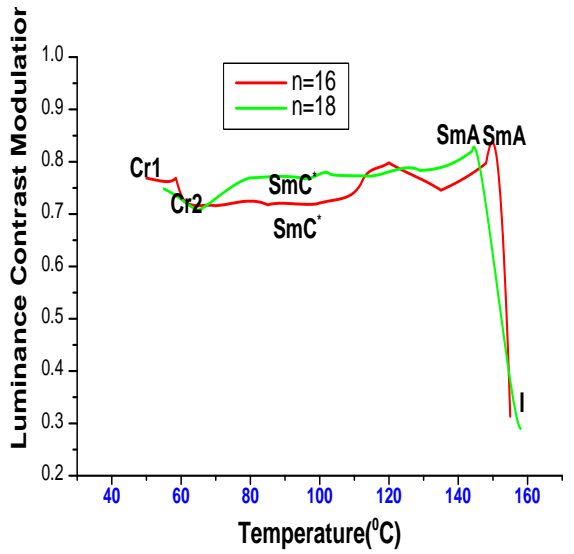

Fig 4. Temperature dependence of luminance contrast modulation for $\mathrm{n}=16$ and 18

Phase transitions of $\mathrm{SmC}^{*}$ and $\mathrm{SmA}$. At isotropic phase LCM steeply drops down to minimum level. So both the samples are suitable for LCD display in this respect.

Gray shades in terms of luminance are calculated from equation 5 and these variations with respective to temperature are observed from fig. 5. Gray shade values keep decreasing in $n=16$ compound however, a sudden shoot up and a drop down in gray shade values are noticed at SmA and isotropic phases respectively. The gray shade values

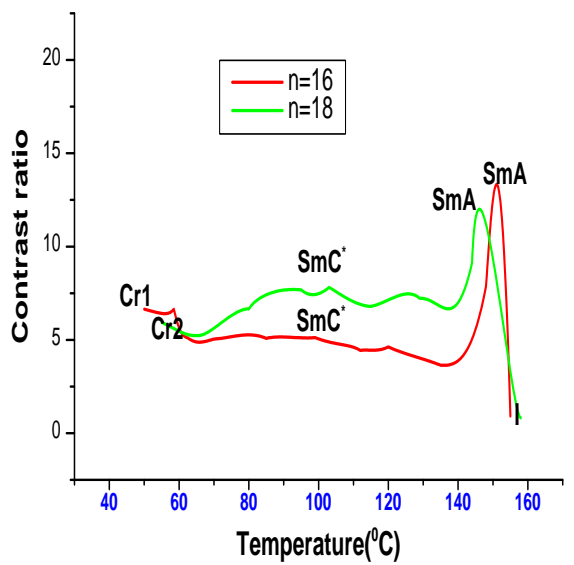

in $\mathrm{n}=18$ compound are increasing uniformly until reaching to SmA phase and later dropping down to isotropic phase. According to the

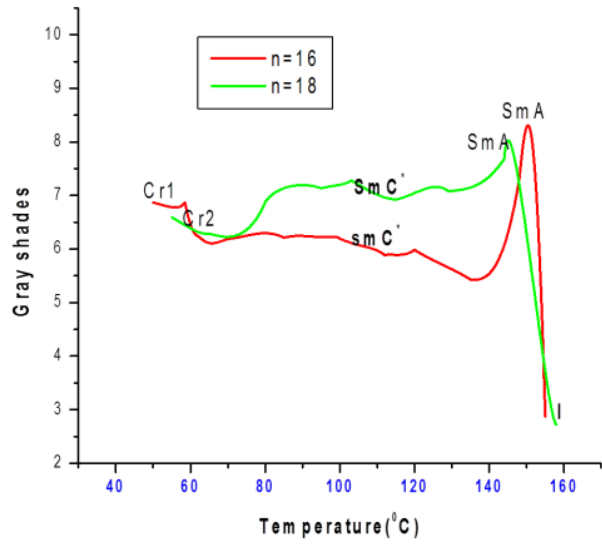

Fig. 5. Temperature dependence of Gray shades for $\mathrm{n}=16$ and 18

literature, the average $\mathrm{G}$ maintained with the samples is indentified around 5.6.The $\mathrm{G}$ for better for both the samples is recorded showing less than standard value of 5.6. Hence, both the samples are not recommended for the LCD panel.

Visual acuity is calculated from equation 6. In the graphs drawn for Luminance versus Visual acuity in fig.6, the visual acuity increases rapidly with the increasing

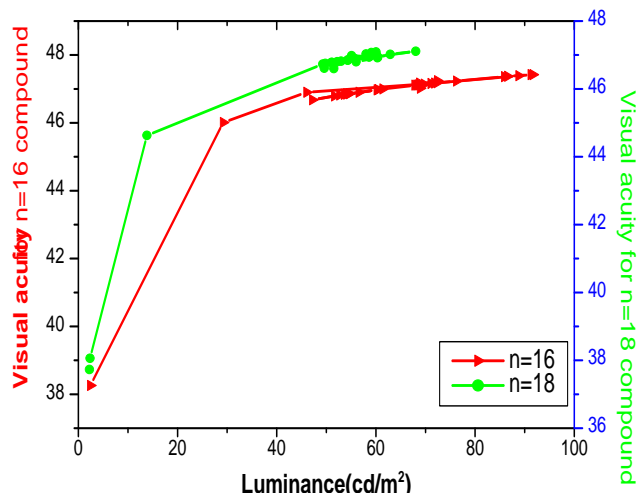

Fig.6. Temperature dependence of visual acuity for $\mathrm{n}=16$ and 18

Of luminance and reaches to saturation at higher values of luminance. In Visual science the term Visual Acuity refers to the ability of an observer to resolve fine pattern in detail. Acuity is usually specified in terms of decimal acuity, defined as the reciprocal of the smallest resolvable, pattern detail in minutes of arc of visual angle, "Normal" or average acuity is considered to be 1.0 (a resolution of $1 \mathrm{~min}$ arc), although many young adults have a decimal acuity slightly better than this [33]. The visual acuity score of an individual is to be expressed as the reciprocal of the angular size of the critical detail within the smallest optotype that can be correctly recognized by the individual. "Clinical visual acuity," is measured in the routine office ophthalmic 
examination. It is defined in this standard as a measurement of the ability to recognize black, highcontrast image on a white background. This measurement is related to visual resolution, but the relationship is a very complex one. Relatively high luminance levels reduce the effects of small variations in luminance. This is desirable for the purpose of comparing visual acuity data [34]. Both the samples are giving good standards of visual acuity at higher luminance. It is drawn that from graph Visual Acuity is more for $\mathrm{n}=18$ compound in comparison to $n=16$. So, to get a better visual acuity levels $n=18$ compound is preferable to $n=16$ in display devices.

Color saturation is calculated from equation 7. The fig.7 shows that the color saturation decreasing for $\mathrm{n}=16$ in $\mathrm{SmC}^{*}$

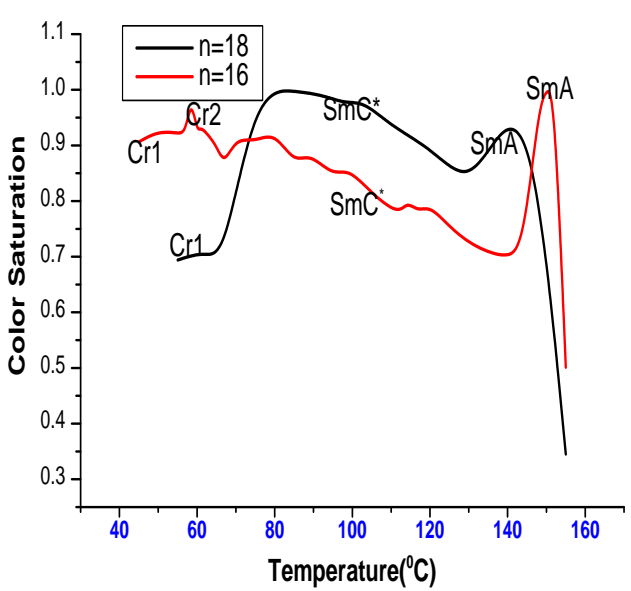

Fig.7. Temperature dependence of colour saturation for $n=16$ and 18

phase and again suddenly shooting up at SmA phase is indicating the more purity of colors at SmA phase, and gray shaded colors for remaining phases. So it is advisable to operate the display at SmA phase with the usage of $n=16$. For $n=18$ almost all colors are pure at $\mathrm{SmC}^{*}$ range and even at SmA Phase. Hence, $\mathrm{n}=18$ is preferable to $\mathrm{n}=16$. Maximum and minimum values of luminance related all parameter for the studied compounds are given in Table 1.

Table 1: Maximum and minimum values of Luminance related optical parameters for $n=16$ and $\mathrm{n}=18$

\begin{tabular}{cccc}
\hline $\begin{array}{c}\text { Optical } \\
\begin{array}{c}\text { Para- } \\
\text { meter }\end{array}\end{array}$ & $\mathrm{SmC}^{*}$ SmA & $\mathrm{SmC}^{*} \stackrel{\text { SmA }}{n=18}$ \\
\hline
\end{tabular}

$\mathrm{T}=155 \mathrm{~K})(\mathrm{T}=152 \mathrm{~K})(\mathrm{T}=155 \mathrm{~K})(\mathrm{T}=146 \mathrm{~K})$

$\begin{array}{ccrcc}\begin{array}{c}\text { Luminance } \\ (\mathrm{cd} / \mathrm{Sqm})\end{array} & 2.43 & 91.00 & 2.22 & 68.00 \\ (140 \mathrm{~K}) & & (139 \mathrm{~K})\end{array}$

$\begin{array}{llllc}\begin{array}{l}\text { Luminance } \\ \text { Contrast Ratio }\end{array} & 1.91 & 20.10 & 1.80 & 19.80 \\ \begin{array}{l}\text { Luminance } \\ \begin{array}{l}\text { Uniformity } \\ \text { Unifrast }\end{array}\end{array} & 0.70 & 4.70 & 0.28 & 0.90 \\ \begin{array}{l}\text { Contrast } \\ \text { Modulation }\end{array} & 0.31 & 0.90 & 0.69 & 6.30 \\ \begin{array}{l}\text { Gray Shades } \\ \text { Visual Acuity }\end{array} & 2.87 & 9.66 & 2.78 & 9.66 \\ & & 46.00 & 35.06 & 47.10 \\ \text { Color } & 0.50 & 1.00 & 0.34 & (158 \mathrm{~K}) \\ \text { Saturation } & & & & 0.99\end{array}$

\section{CONCLUSION}

Temperature dependence of different optical parameters of FLC compounds are computed through image analysis technique. The investigated luminance dependant display device parameters are found in good agreement with the literature values. From the present study both the samples are not suitable for the LCD panel, because $n=16$ and $n=18$ samples are showing maximum luminance dependent parametric values at $\mathrm{SmA}$ phase instead of $\mathrm{SmC}^{*}$ phase. This kind of image analysis technique for measurement of luminance and its dependent parameters replaces the various instrumental operating techniques like Spectroradiometer, spectrophotometer, etc.,

\section{ACKNOWLEDGEMENTS:}

The Authors M.S and SSS would like to thank UGC, New Delhi for providing -BSR Meritorious Research Fellowship (Letter No. F.251/2014-2015).and BSR Faculty fellowship No.F.181/2011(BSR) Dt: 4/01/2017 respectively. The authors also gratefully acknowledge University Grants Commission Departmental Special Assistance at Level I program No. F.530/1/ DSA- 1/2015 (SAP1), dated 12 May 2015, Department of Science and Technology-Fund for Improving Science and Technology program No.DST/FIST/ PSI-002/2011 dated 20-12-2011, New Delhi, to the department of Physics, Acharya Nagarjuna University for providing financial assistance.

\section{REFERENCES}

[1]. Ferroelectric liquid crystal displays (FLCD) www.slideshare.net Published on Apr 03, 2014, seen on June 12, 2014.

[2]. S.Singh, Liquid Crystals: Fundamentals. (Singapore: World Scientific Publishing Co. ,2002).

[3]. D.Demus, G.W. Goodby J, Gray, H W Spiess, V.Vil, Physical Properties of Liquid Crystals. (Singapore: Wiley- VCH Publishing Co; 1999).

[4]. S.Chandra Sekhar, Liquid Crystals. (New York: Cambridge University Press; 1992). 
[5]. P.Oswald, PPieranski The Liquid Crystal Books Series: Smectic and Columnar Liquid Crystals - Concepts and Physical Properties Illustrated by Experimens. (London: CRC Press, 2006).

[6]. I C Khoo, Liquid Crystals, (New Jersy : Hoboken Wiley- Interscience Publishing Co; 2007).

[7]. R B Meyer, Ferroelectric liquid crystals: a review. Mol Cryst Liq Cryst. 40(14),1977, 33-48.

[8]. R B Meyer,L Liebert, L Strzelecki, P Keller. Ferroelectric liquid crystals: a review. Phys Chem Lett., 36,1975, 69-71.

[9]. J W Goodby, E Chin, J M Geary, J S Patel, $\mathrm{P}$ L Finn, The ferroelectric and liquidCrystalline properties of some chiral alkyl 4-n-alkanoy loxy biphenyl 4_-carboxylates J Chem Soc, Faraday Trans. 83(11), 1987,3429-3446.

[10]. S T Ha, G Y Yeap, P L Boey, synthesis and smectogenic $A$ and $C^{*}$ properties of $(\mathrm{S})-$ (-)-2-methylbutyl4- (4-n- alkanoyloxy benzoy loxy) bipheny 1-4- carboxylates. Internat J Phyl Sci. 5(3),2010,182-191.

[11]. S S Sastry, B Gowri Sankara Rao, H S Tiong, S.Lakshminarayana. Identification of phase transitions temperatures by statistical image analysis. Liq Cryst. 39(6),2012,695700

[12]. B. Montrucchio, A.Sparavigna, A Strigazzi. A new image processing method for enhancing the detection sensitivity of smooth transitions in liquid crystals. Liq Cryst.,24(6), 1998,841-852.

[13]. A. Sparavigna, A. Mello B. Montrucchio, Texture transitions in the liquid crystalline alky loxybenzoic acid 6OBAC. Phase transitions, 79(4-5), 2006, 293-303.

[14]. J Eccher, AR Sampaio, RC Viscovini, G Conte, E Westphal, H Callardo, IH Bechtold, Image processing as a tool for phase transitions identification. $\mathrm{J}$ Mol Liq.153(2-3), 2010,162-166.

[15]. D Wouller, H Gabele. The usage of digital cameras as luminance meters. Proc. SPIEIS\&T,6502,2007,1-11.

[16]. G Yulia, P Tania, R Erik, S.Hans- Peter. Sky based light metering for high dynamic range images. Pacific Graphics, 33 (7), 2014,1-9.

[17]. S.S.Sastry. B Gowri Sankara Rao , KB Mahalakshmi, K Mallika, C. Nageswara Rao. S T Ha, Image Analysis Studies for Phase Transitions of Ferroelectric Liquid Crystals. ISR Cond Mat Phys. 2012,1-8.

[18]. RC Gonzalez, RE Wood, SL Eddins Digital image processing using MATLAB.
(Dorling Kindersley (India) Pvt. Ltd., Delhi,2004

[19]. RC Gonzalez, $P$ Wintz.Digital Image Processing- Reading. (USA :Mass, AddisonWesley Co., 1987).

[20]. V Karthikeyani, K Duraiswamy, P Kamalak kannan. Conversion of Gray-scale image to Color Image with and without Texture Synthesis. IJCSNS,7(4),2007.

[21]. B Bahadur. Liquid Crysta Applications and Uses.(Singapore: World Scientific Pub. Co., 1991).

[22]. Y Peter. Ngai. The relationship between Luminance Uniformity and Brightness Perception. J Illuminating Engg Soc. Winter 2000, 41-50.

[23]. B Mathew, EV Micheal, C dayle jean, Hand book of Human factor. in medical device design. (USA :CRC press, 1992).

[24]. T Shinya, A Hiroshi Yak, H Norimak, N Yasuhiko, M Yoshio, Akiko Hattori. A preliminary study for exploring the luminance ratio of liquid-crystal displays required for display of radiography. Radio Physics Technology, 7,2014,73-78.

[25]. A Kenneth Fetterly, R B Hartwig, J Michael Flynn, Ehsansamei. Introduction to Grayscale Calibration and Related Aspects of Medical Imaging Grade Liquid Crystal Displays. J Digit imaging, 21(2),2008,193207.

[26]. Boff, Kenneth RandLincoln, E Janet. Human perception and performance ,(USA: Department of Energy, 1988,908).

[27]. T Nelson, II JR Wullert. Electronic information display technologies.(Singapore :World Scientific Publishing Co., 1997).

[28]. Martin helander. A guide to human factors and ergonomics.(Florida: CRC press, 2006).

[29]. T Delphine, L Odile Brosseau,F Jocelyn, V D François. Maturation of the sensitivity for luminance and contrast modulated patterns during development of normal and pathological human children. Vision Research, 47(12), 2007,1561-1569.

[30]. J A Jacko. The Human-Computer Interaction: Handbook, Fundamental, Evolving Technologies, and Emerging Applications. (USA,CRC Press. 2007).

[31]. J A Castellano, Handbook of Display Technology.( San Diego : Academic Press, 1992).

[32]. S Shaler. The relation between visualacuity and illumination. J Gen Psychology, 11(3), $1928,255-2$

[33]. G.Ward Larson, H Rushmeier, C.A.Piatko visibility matching tone reproduction 
operator for high dynamic range scenes. IEEE TVCG, 3(4), 1997,291-2.

[34]. R B Kenneth,G.Janet E L,Harry , Human Perception and Performance, (Ohio: Armstrong Aerospace Medical Research Laboratory WrightPatterson Air Force Base; 1988). 80.82), NASA, Ads, Researcher Id Thomson Reuters, DOAJ.

S. Sreehari Sastry. "Computation of Liquid Crystal Display Device Parameters Through Image Analysis." International Journal of Engineering Research and Applications (IJERA) 7.7 (2017): 24-31. 